CCL18 levels also positively correlated with DAS-28 score at baseline $(P=0.003)$. CCL18 and CXCL16 levels did not correlate with joint damage or radiographic disease progression. Levels of CCL18 decreased significantly $(P<0.0001)$ after initiation of infliximab treatment, independently of disease activity.

The authors conclude that, while CCL18 correlated less strongly with disease activity than erythrocyte sedimentation rate and C-reactive protein level, further research into the roles of CCL18 and CXCL16 is required to understand their roles in $\mathrm{RA}$.

Original article van Lieshout AWT et al. (2007) Circulating levels of the chemokine CCL18 but not CXCL16 are elevated and correlate with disease activity in rheumatoid arthritis.

Ann Rheum Dis 66: 1334-1338

\section{Variation in STAT4 linked with RA and SLE}

A region of chromosome $2 q$ has previously been linked with susceptibility to rheumatoid arthritis (RA) in families of European descent. Remmers et al. conducted a case-control disease-association analysis of 13 candidate genes in this RA-linked region. They found that a single-nucleotide polymorphism (SNP) in the STAT4 gene (which encodes a transcription factor that mediates the effects of both interleukin [IL]-12 and IL-23) was associated with both RA and systemic lupus erythematosus (SLE).

SNPs were tested for association with RA in 1,620 North American patients of European descent with established RA and 2,635 matched controls, and also in 1,529 patients with early $\mathrm{RA}$ and 881 controls from Sweden. Four SNPs in the third intron of STAT4 had strong associations with RA $(P<0.001)$; the most significant association was with the rs7574865 SNP $(P=0.0000829)$. The minor allele of this SNP was present in $27 \%$ of North American and $25 \%$ of Swedish patients, compared with $22 \%$ of controls.

Because the region containing STAT4 has also been linked with SLE, the association of rs7574865 was also tested in three groups totaling 1,039 patients with SLE and 1,248 controls. The minor allele was present in $29-31 \%$ of patients compared with $22-23 \%$ of controls. Homozygosity of this allele is associated with an odds ratio of 1.61 for RA and 2.41 for SLE. The association with RA has since been replicated in a Korean cohort.

Original article Remmers EF et al. (2007) STAT4 and the risk of rheumatoid arthritis and systemic lupus erythematosus. N Engl J Med 357: 977-986

\section{A tyrosine kinase inhibitor successfully treats RA in rats}

The response to immune-complex signaling, mediated through Fc receptors, has been implicated in the development of rheumatoid arthritis (RA). Spleen tyrosine kinase (Syk) is a critical component in immune-complex-mediated signal transduction. Pine et al. evaluated the anti-RA activity of R406, a small-molecule Syk inhibitor, in a rat model of RA. R406, or its prodrug R788, suppressed bone erosion and joint inflammation in rats with collagen-induced arthritis.

A severe clinical arthritis developed in the rats 10-11 days after injection of type II collagen. When inflammation was observed in at least one hind paw (clinical arthritis score $=1$ ), twice-daily administration of R406 (10 or $30 \mathrm{mg} / \mathrm{kg}$ ) or control commenced. In control animals, mean arthritis score peaked at $5.6 \pm 0.5$, and remained unchanged for the 18 day duration of the study. The $30 \mathrm{mg} / \mathrm{kg}$ dose of R406 resulted in complete remission of arthritis by study end (arthritis score $0.1 \pm 0.1$ ); the $10 \mathrm{mg} / \mathrm{kg}$ dose resulted in delayed onset and reduced severity of RA compared with vehicle. The prodrug R788 also reduced RA severity. Radiographs of hind paws showed significant protection against bone erosion by R406 and R788 versus vehicle. Cytokine levels elevated in arthritic rats were completely suppressed by R406, and the half-life of anti-tumor necrosis factor antibodies was significantly increased by co-administration of R406.

The authors conclude that inhibition of Syk with R406 can suppress bone erosion and inflammation in RA, and can also extend the half-life of anti-RA biological agents. Phase II clinical trials of R406 are ongoing.

Original article Pine PR et al. (2007) Inflammation and bone erosion are suppressed in models of rheumatoid arthritis following treatment with a novel Syk inhibitor. Clin Immunol 124: 244-257 